\title{
Extending a Lippmann style seismometer's dynamic range by using a non-linear feedback circuit
}

\author{
G. Romeo and G. Spinelli \\ Istituto Nazionale di Geofisica e Vulcanologia, Roma, Italy \\ Correspondence to: G. Romeo (giovanni.romeo@ingv.it)
}

Received: 23 May 2013 - Revised: 24 July 2013 - Accepted: 5 August 2013 - Published: 11 October 2013

\begin{abstract}
A Lippmann style seismometer (Lippmann and Gebrande, 1983) uses a single-coil velocity-feedback method in order to extend toward lower frequencies a geophone's frequency response. Strong seismic signals may saturate the electronics, sometimes clipping the signal or producing the characteristic whale-shaped recording.

Adding a non linear feedback in the electronic circuit may avoid saturation, allowing the strong-motion use of the seismometer without affecting the usual performance. Such a seismometer will allow unsaturated data in epicentral area while offering nice low signal recording for far events.
\end{abstract}

\section{Introduction}

Strong seismic signal easily saturates sensitive force-balance velocimeters. So seismic networks in seismically active areas may suffer from blindness, around the epicentral areas, because of sensors saturation. This difficulty is usually overcome by using a double set of instruments (a sensitive seismometer and a strong motion seismometer) to extend the station's dynamic range.

It is possible to obtain the same effect (the dynamic range extension) using a double feedback circuit in a single instrument (INGV's patent pending), one for weak and one for strong signals. This obviously decreases the station's cost. The article shows the double feedback strategy applied to a Lippman-style seismometer.

\section{Principle of operations}

There are two effects that bring a seismometer to saturation. A mechanical effect (the mass displacement exceeds the allowed span) and an electrical effect (the electric signals are

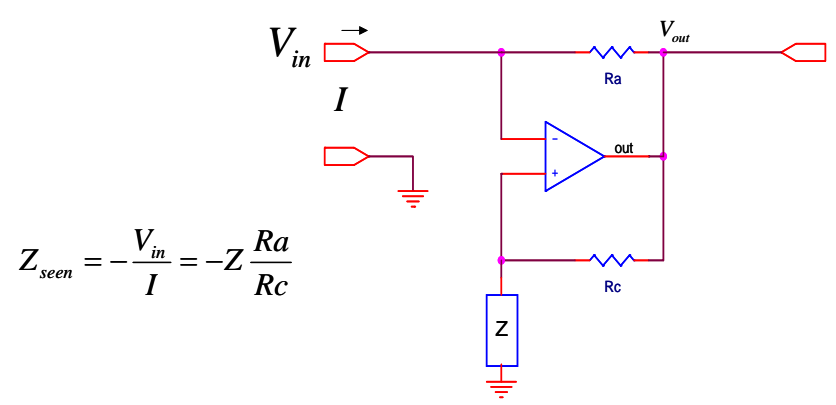

Fig. 1. A Lippmann seismometer front-end is built using a NIC (Negative Impedance Converter) (Horowitz and Hill, 2006). The input impedance of this circuit, $Z_{\text {seen }}$, is negative. This allows overdamping an electromagnetic seismic transducer that is the basis of the Lippmann idea.

too high for the electronic circuit embedded in the seismometer). The electrical front-end of a Lippmann seismometer is depicted in Fig. 1

The basic idea of the Lippmann seismometer is to actively over-dampen an electromagnetic seismometer. Briefly (and roughly) the skeleton of a Lippmann-style seismometer is contained in the following four points:

- Overdamping a seismometer means stopping the mass (even if the stopping is caused by the mass velocity instead of by the mass position).

- With a very high overdamping the mass moves very little.

- If the mass has been damped using an electromagnetic actuator the current through the motor coil is proportional to the force needed to stop the mass and, in case of strong over-damping, to the ground acceleration. 


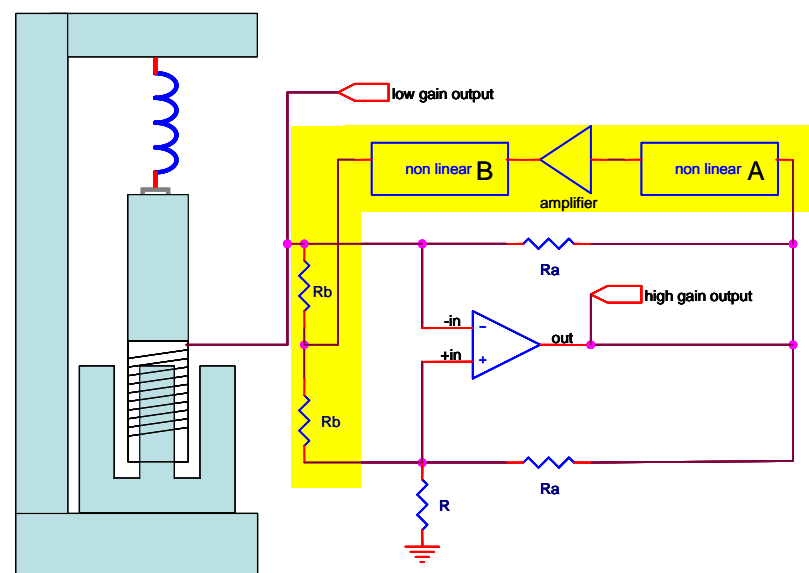

Fig. 2. The double-feedback seismometer. The area highlighted in yellow is the one added to obtain the strong feedback. In case of weak signals the strong feedback circuit is not engaged (because of the non linear blocks), and the only differences respect to the fig 1 circuit are the two resistors $R_{\mathrm{b}}$. Since the operational amplifier is used as nullor, and there is not voltage between -in and +in, these resistors have no effect until the non linear blocks are engaged, although they decrease the noise performance (Horowitz and Hill, 2006).

- The ground velocity may be calculated by integrating the voice coil current.

An over-damped seismometer cannot saturate mechanically (if the mass is stopped by the feedback force its displacement cannot reach the mechanics limits), but only electrically (the current supplied to the motor coil is not enough to compensate strong accelerations). In this case a mechanical saturation may happen, caused by insufficient feedback force.

A geophone's damping factor may be written as (Romeo and Braun, 2007):

$\xi=\frac{G^{2}}{2\left(R+R_{\text {coil }}\right) \omega_{0} M}$

where $G$ is the pick-up coil gain (Volt/velocity), $\xi$ is the damping factor, $M$ the mass, $\omega_{0} / 2 \pi$ the undamped resonance frequency and, $R$ the damping resistor across the transducer coil and $R_{\text {coil }}$ the transducer coil resistance.

If we assume a geophone directly connected to the Fig. 1 NIC $V_{\text {in }}$ is the voltage across the pick up coil and $R$ becomes $Z_{\text {seen }}$. The use of the NIC is mandatory to tune $R+R_{\text {coil }}$ to the wanted value (usually lower than $R_{\text {coil }}$ ).

The maximum current that we may force through the pickup coil is given by $V_{\mathrm{pwr}} /\left(R_{\mathrm{a}}+R_{\text {coil }}\right)$.

So, ideally, the maximum acceleration we may produce using the pick-up coil is:

$a=\frac{G \cdot V_{\mathrm{pwr}}}{\left(R_{\mathrm{a}}+R_{\mathrm{coil}}\right) M}$

where $V_{\text {pwr }}$ is the NIC supply voltage and $R a$ is the feed-back resistor. So $V_{\mathrm{pwr}}$ and $R_{\mathrm{a}}$ are two electrical values we may tune to control the maximum acceleration the seismometer can handle.

\section{Double feedback recipe}

The recipe goal is to provide a strong feedback able to handle close seismic events without affecting the high sensitivity seismometer performance. This is obtained using the configuration shown in Fig. 2.

The circuit has an amplifier connected using two non linear blocks $\mathrm{A}$ and $\mathrm{B}$.

Both blocks have the same comportment (Fig. 3), but it is mandatory for the block B to offer a high output impedance in case of low voltage output. The non linear blocks make the additional feedback to electrically disappear in case of weak signals, and to be engaged in case of strong signals. Figure 2 shows two outputs, called hi gain output and low gain output. In case of weak signal they output the same signal with a different scale factor (because of the operational amplifier gain). In case of strong signal the low gain output keeps the linear response while the high gain output is clipped.

Figure 4 shows the non linear feedback front end stimulated by an acceleration signal. The picture has been obtained by mean of a pSPICE S13 model (Geothech Instruments, 2000) (Romeo, 2012).

The conclusion of the process in a Lippmann seismometer is the integration of the NIC front end to obtain the ground velocity. The non linear front end supplies two outputs. These may be handled individually, producing two separated set of data or, better, digitally combined in one single high-dynamic data stream.

\section{Feeding the non-linear feedback seismometer with a real event}

A real event record has been used to check the seismometer's model with a real stimulus. We used records from the station MIR06 in Pieve di Coriano, Italy, (45.050115 11.0874616) operating a Le-5s and an Episensor. The event chosen (29 May 2012, 06:57:00 UTC) was strong enough to saturate the Le-5s leaving the Episensor in linear condition. When working in range the instruments show compatible recordings. Figure 5 shows the two recordings, comparing two unsaturated data segments to verify the instruments congruence. Figure 6 shows some output of the non linear feedback seismometer stimulated with the Pieve event. On the left diagram the velocity output, obtained by applying a 1 pole low pass to the low gain output. The seismogram appears unsaturated. On the right diagram the mass displacement does not exceed $\pm 0.3 \mathrm{~mm}$. 


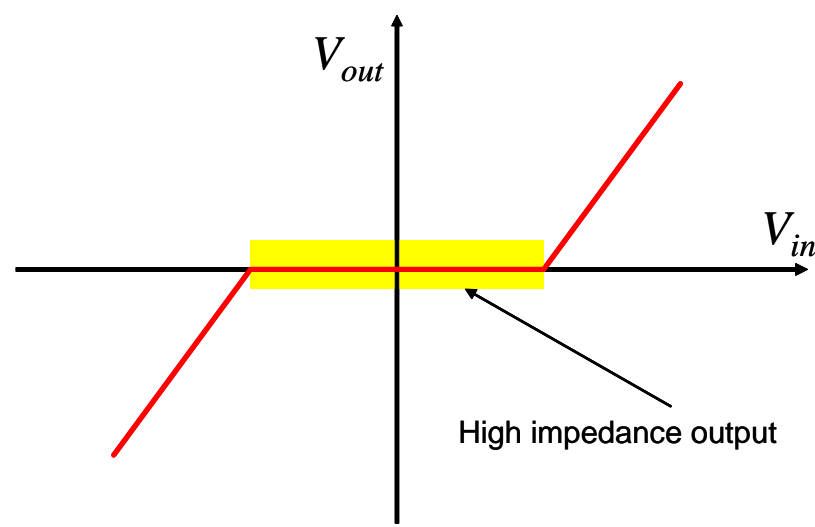

Fig. 3. Both A and B blocks have the same $V_{\text {out }}$ vs. $V_{\text {in }}$ characteristics; the only difference is that the block B must have high output impedance in case of low voltage (yellow area).

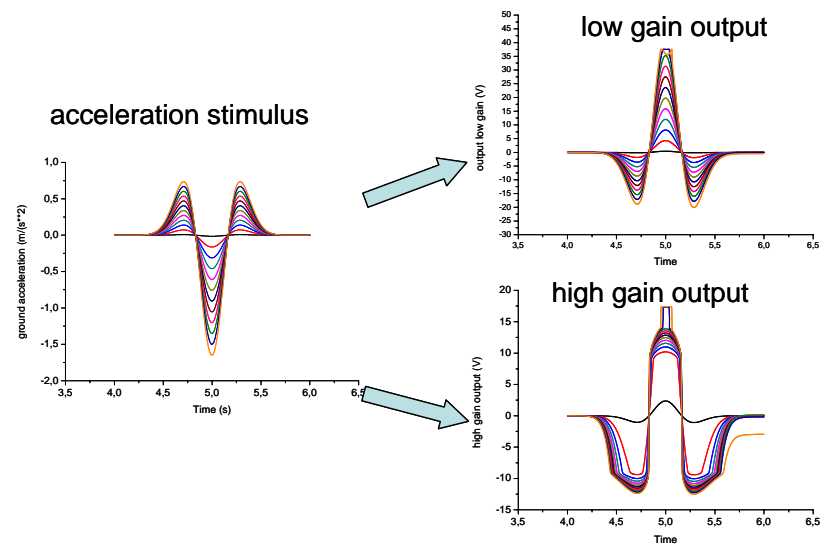

Fig. 4. We used a variable amplitude acceleration stimulus to check the comportment of the non linear front-end, using, as transducer, an s13 geophone (Geothech Instruments, 2000). Referring to Eq. (2) $V_{\text {pwr }}$ and $R_{\mathrm{a}}$ were respectively $45 \mathrm{~V}$ and $100 \mathrm{Ohm}$. The low gain output shows a linear response until $0.15 \mathrm{~g}$, while the high gain output stops being linear at, roughly, $0.01 \mathrm{~g}$.

\section{Conclusions}

Double feedback seismometers combine broad-band and strong-motion characteristics and may be a cost effective solution to improve seismic network performance in active areas. We explored the possibility to extend the operating range of a Lippman seismometer using a secondary feedback circuit, operating only in case of strong signal. Although some circuital solution adopted may decrease the noise performance, simulation results and lab tests showed the strategy may work, and we are going to complete the instrument evaluation with a field test.

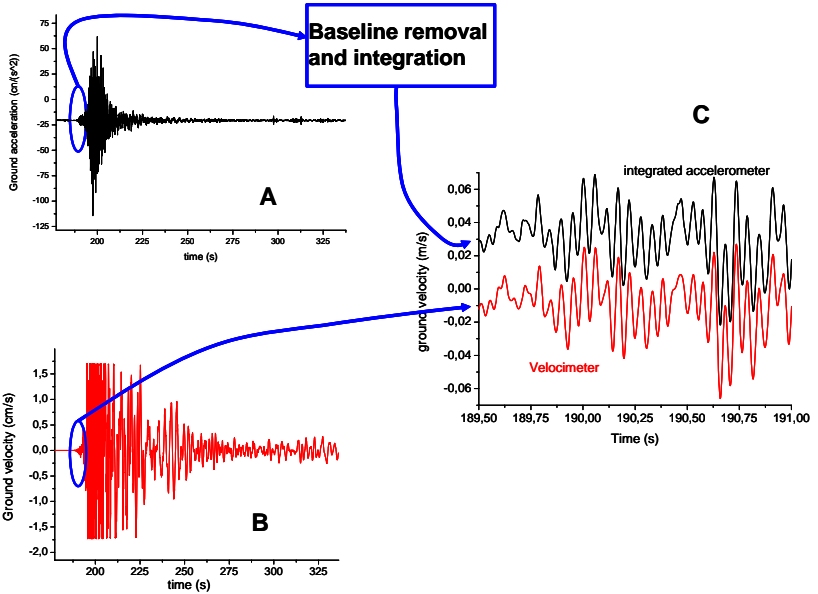

Fig. 5. The event of Pieve di Coriano recorded by the Episensor (A) and the Le-5s (B). The Le-5s recording shows saturation. After operating offset removal and integration on the accelerometer's signal, the two instruments show a nice agreement $(\mathbf{C})$. This agreement disappears for strong signals, even before the velocimeter's clipping. The congruence of signals makes confident that we may use the integrated accelerometer signal as velocity source to feed the models to study the comportment of the double-feedback seismometer in a real case.
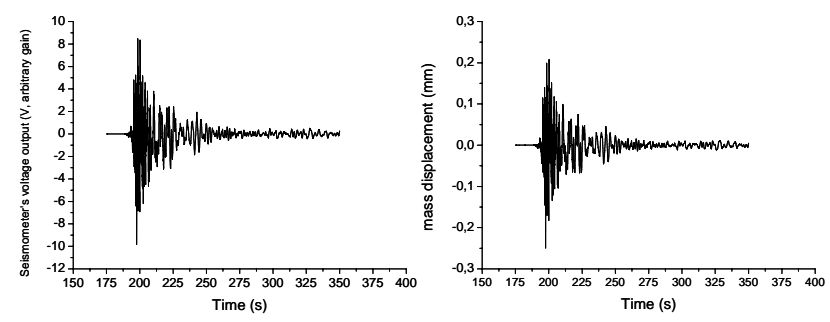

Fig. 6. On the left the Pieve di Coriano event obtained from the low gain output. On the right the mass displacement.

Acknowledgements. The authors thank Paolo Benedetti for helping in building the prototype, Giuliano Milana, for providing the data used in the simulations, and the referees for the precious suggestions.

\section{References}

Geothech Instruments LLC. PORTABLE SHORT-PERIOD SEISMOMETER MODEL S-13 - Operation and maintenance manual, http://www.geoinstr.com/pub/manuals/s-13.pdf, 2000.

Horowitz, P. and Hill, W.: The art of electronics, Cambridge University Press, 2006.

Lippmann, E. and Gebrande, H.: Eine einfache Methide zur Erweiterung des Meßereichs Elektrodynamischer Seismometer, Annual meeting of the German Geophysical Society, Aachen, 1983.

Romeo, G.: Whale watching: effects of strong signals on Lippmann style seismometers, J. Seismol., 16, 25-34, 2012. 
Romeo, G. and Braun, T.: Appunti di sismometria, Quaderni di Geofisica, 46, (http://portale.ingv.it/portale_ingv/ produzione-scientifica/quaderni-di-geofisica/archivio/ quaderni-di-geofisica-2007), 2007. 